149 THE POTENTIAL FOR VIRTUAL REALITY THERAPY IN PALLIATIVE CARE - PRELIMINARY FINDINGS

Letizia Perna-Forrest, Ollie Minton. Royal Trinity Hospice

10.1136/bmjspcare-2019-ASP.172

Background Researchers have long been interested in the physiological and psychological aspects of wellbeing. Studies have found that Virtual Reality (VR) therapy, using computer generated environments and avatars, can have positive effects in relieving pain in some patient populations and can be used to alleviate symptoms of depression, anxiety, and Post Traumatic Stress Disorder. To date, there is little published research about the physical and psychological impact of VR therapy using real life environments/settings and the potential for VR to be used effectively in palliative care has not been fully investigated.

Method Participants are given a single VR therapy experience lasting no more than $4 \mathrm{~min}$, once a week, for 4 weeks. Quantitative data is obtained through the comparative analysis of pre- and post-session Edmonton Symptom Assessment System: Revised (ESAS-R) scores and qualitative data is gathered through set interviews completed after each VR therapy.

Results Preliminary data evidences a 53\% mean reduction in pain symptoms, $66 \%$ mean reduction in fatigue, $60 \%$ mean reduction in drowsiness, $50 \%$ mean reduction of SOB, $52 \%$ mean reduction in depression, and $62 \%$ mean reduction in anxiety with an overall $49 \%$ mean increase in overall wellbeing. Qualitatively, participants spoke about feeling more relaxed and generally 'happier' as a result of VR. Additionally participants spoke about VR connecting them to positive memories as well giving them a sense of freedom both from their illness, their symptoms, and life as a patient.

Conclusion Preliminary findings positively demonstrate a reduction of common physiological and psychological palliative care symptoms. Additional participants and VR therapy sessions are planned. Additional positive results will provide robust evidence for VR Therapy to be adopted and used alongside current symptom control measures used in palliative care.

\section{UNDERSTANDING HOW DECISIONS TO PRESENT TO THE EMERGENCY DEPARTMENT ARE MADE BY PATIENTS WITH ACUTE-ON-CHRONIC BREATHLESSNESS, THEIR FAMILY CARER AND CLINICIAN}

A Hutchinson, C Barbetta, K Galvin, M Twiddy, MJ Johnson. University of Hull, Ospedale S. Maria degli Angeli, Pordenone, Italy and University of Brighton

\subsection{6/bmjspcare-2019-ASP.173}

Background Chronic breathlessness is common in cardiorespiratory conditions and is frequently associated with emergency department (ED) presentation when the patient experiences acute-on-chronic breathlessness. Breathing Space is a concept combining patient coping, help-seeking and clinician responsiveness to breathlessness in addition to disease-management. This study aimed to explore whether Breathing Space may help explain the decision by patients with acute-on-chronic breathlessness to present to the ED.

Methods Secondary mixed-methods analysis of patient selfreport survey, case note and interview data. We used an indepth case-history approach to synthesise these source data from eight representative patients who presented to the ED due to acute-on-chronic breathlessness. Patient-interview data were also linked with interview data from their family carers and clinicians.

Results Secondary analysis of eight survey and case note reviews, combined with analysis of eight patient interviews (four with a carer) and six clinician interviews was conducted. The Breathing Space concept was useful in understanding the individual patient's decision to attend the ED. Having a clinician who both understands the impact of breathlessness on their patient and offers ways of managing breathlessness in addition to treating the underlying disease seems to be important. Clinicians responsive to breathlessness were able to encourage a disengaged patient (with restricted Breathing Space) to move towards a more adaptive way of living with breathlessness (greater Breathing Space). The converse was seen when clinicians had a sole focus on disease-directed treatment.

Conclusion The Breathing Space concept may be useful for clinicians caring for people with chronic breathlessness. Early assessment and management of the Breathing Space of the patient (and carer) may help improve the patient's (and carer's) quality of life and the management of acute-onchronic breathlessness crisis.

\section{SYMPTOM MANAGEMENT IN END STAGE LIVER DISEASE - RESULTS OF A REGIONAL PALLIATIVE CARE NETWORK SURVEY}

G Ting, D Foster, S Fradsham, J Henry, J Hill, S Howarth, L Owens, K Rugen, A Khodabukus. Royal Liverpool and Broadgreen University Hospitals NHS Trust, Marie Curie Hospice Liverpool, Aintree University Hospital NHS Foundation Trust, Woodlands Hospice

\subsection{6/bmjspcare-2019-ASP.174}

Background Symptom management in patients with end-stage liver disease can be challenging due to hepatic dysfunction. The aim of this study was to scrutinise the literature and current practices among healthcare professionals leading to the development of regional standards and guidelines.

Method A systematic review of the literature was undertaken to look for evidence of best practice in the management of common symptoms seen at the end of life in this patient cohort. In addition, this review assessed patient experience of living and dying with end-stage liver disease. To evaluate local clinical practices and professional needs, healthcare professionals in the Mersey and Cheshire regional palliative care network were surveyed. This survey was supplemented by a retrospective case note review of patients with end-stage liver disease known to palliative care teams in the hospital, hospice and community.

Results The literature review found little robust evidence for managing commonly encountered symptoms in patients with end-stage liver disease at the end of life. Fifty-one healthcare professionals (31 doctors, 19 nurses, 1 pharmacist) were surveyed. The majority of responders highlighted a need for upto-date training in pharmacology specific to end-stage liver disease. Midazolam was most frequently prescribed to manage agitation. Forty-eight case notes were reviewed. Twenty-six (54\%) patients had evidence of hepatic encephalopathy at the time of their admission. In 38\% of the cases, Lactulose was used and in $23 \%$ of the cases, benzodiazepines were used. In $88 \%$ cases, terminal agitation was managed with Midazolam and in $15 \%$ cases, Levomepromazine was used. 
Conclusion There is clearly a need for greater awareness of the pharmacology in symptom control in end-stage liver disease. As a result of this study, peer-reviewed clinical standards and guidelines are being formulated for the management of specific symptoms related to end-stage liver disease to provide standardised care across the network.

\section{PLANT: 'PALLIATIVE LIFE-STATE \& NUTRITION TOOL' ๑ - REVIEW OF THE USE OF PLANT IN A HOSPICE}

Eleni Tsiompanou. Woking and Sam Beare Hospices, Health Being Institute, College of Medicine

\subsection{6/bmispcare-2019-ASP.175}

Background Palliative patients have a number of dietary concerns. The first step to good nutrition care is nutrition assessment. The PLANT tool, developed with the support of a multidisciplinary team, highlights problems that affect the intake, digestion and assimilation of food and overall quality of life. It has been offered to the community and in-patient unit teams of a hospice for use.

Methods a restrospective review of 30 randomly chosen PLANT tools filled in the last 6 months was done. Hospice staff were asked to give feedback about its' use.

Results $13 \%$ of patients had special dietary requirements and $13 \%$ food allergies and intolerances. $77 \%$ had lost weight while $23 \%$ had gained weight. $70 \%$ of patients were concerned about their weight. In $73 \%$ of cases current weight was recorded, while BMI was calculated in only $33 \%$.

Symptoms in order of frequency were: fatigue 100\%, dry mouth $87 \%$, sadness or depression $87 \%$, reduced appetite $83 \%$, flatulence $83 \%$, sleep problems $80 \%$, constipation $77 \%$, taste or smell changes $73 \%$, nausea $73 \%$, abdominal bloating $67 \%$, abdominal pain $63 \%$, belching $63 \%$, sore mouth/throat $60 \%$, hiccups $60 \%$, vomiting $53 \%$, loneliness or isolation $53 \%$, diarrhoea 43\%, nocturnal defaecation 43\%, heartburn/ acid reflux $33 \%$.

Hospice healthcare professionals found the tool innovative and useful. However, some thought it was inappropriate to ask palliative care patients about weight and eating related symptoms and voiced their concern that it would cause unnecessary distress to patients. Furthermore, some of the staff thought that it was adding to their workload by having to fill in yet another tool.

Conclusions The PLANT tool can help to identify key symptoms that affect the nutritional state and overall life-state of patients. More work is needed to increase awareness amongst healthcare professionals of the impact of nutrition problems on patients' health, disease and sense of wellbeing.

\section{AUDIT OF COMPLIANCE WITH NICE GUIDANCE ON PRESCRIBING STRONG OPIOIDS}

Tammy Oxley, Lucy Walker, Sophie Thomas, Yasmin Rejaei, Debbie Atkinson. Mid Yorkshire NHS Hospital Trust

\subsection{6/bmjspcare-2019-ASP.176}

The 2012 NICE guidelines on prescribing strong opioids cover prescribing in advanced disease. The aims of our audit were to demonstrate compliance with these guidelines at The Mid Yorkshire Hospitals NHS Trust (MYHT).
A prospective audit was undertaken and data collected from 97 patients who had been started on strong opioids across MYHT. Patients included were those reviewed by the hospital and community Specialist Palliative Care Team (SPCT) and inpatients on both respiratory and oncology wards. Patients reviewed by the SPCT team were identified using the weekly patient MDT list and further information found using SystemOne. Hospital inpatients were identified using the controlled drug books and cross referencing with medical notes.

$48 \%$ of patients were initiated on a strong opioid without having previously been on a weak opioid. Information giving was poor with only $0 \%-20 \%$ of the audited patients being informed about specific matters documented in the NICE guidelines, including advice regarding drowsiness and driving. Disappointingly, only $42 \%$ of patients commenced on a strong opioid were also prescribed a laxative. Prescribing in renal impairment was not fully in line with NICE guidance suggesting consultation with a specialist if moderate to severe impairment present - in 4/6 cases where inpatients (excluding those reviewed by the SPCT) had an eGFR of $<30$ no specialist input was sought.

Limitations of the audit include that a relatively small data set was collected from each clinical area. In addition, the audit relied on healthcare professionals formally documenting decision making and discussions with patients in the notes.

Recommendations include sharing the results with the audited specialities and specific education regarding the benefits of using a trust-wide opioid leaflet to assist with information giving. A quality improvement project focusing on improving co-prescribing laxatives was subsequently undertaken within the respiratory department.

\section{HYPONATREMIA IN PALLIATIVE CARE; IS IT ALWAYS SIADH?}

Abraham M Varghese, Sunitha M Daniel. Little Flower Hospital and Research Centre Angamaly, Kerala, India, Amrita Institute of Medical Sciences, Amrita University, Kochi, India

\subsection{6/bmjspcare-2019-ASP.177}

Background Hyponatremia, the most common electrolyte disturbance in clinical practice affecting up to $15 \%-28 \%$ of admitted patients, is defined as a serum sodium concentration $([\mathrm{Na}+])<135 \mathrm{mmol} / \mathrm{L}$. Hyponatremia at admission is known to increase the duration and cost of stay of hospitalized patients. The prevalence of hyponatremia in palliative care is about $28 \%$ of at the time of referral. Hyponatremia can be asymptomatic or can cause symptoms like nausea and lethargy to convulsions and coma. It is important to evaluate the cause of hyponatremia to have a targeted management strategy.

Methods A retrospective case note analysis was carried out among patients admitted under a palliative care unit of a tertiary referral hospital in South India, over a period of 6 months. The sodium level on admission and during the inpatient stay, along with symptoms, drug history and cause of hyponatremia was evaluated.

Results Out of 11 patients who presented with hyponatremia, median age was 59;7 patients were male. All the patients had a cancer diagnosis, with $28 \%$ had cancer of GI tract,91\% had stage IV disease. The median sodium level on admission was $125 ; 82 \%$ had hypotonic hyponatraemia. 37\% were asymptomatic on presentation, while $18 \%$ had generalised weakness. 